DR. AYAKO SUZUKI (Orcid ID : 0000-0003-1824-1067)

3

4

Received Date : 30-Apr-2016

6

Revised Date : 29-Dec-2016

7

Accepted Date : 20-Jan-2017

8

Handling Editor: Raúl Andrade

\title{
Associations of Gender and a Proxy of female Menopausal status with Histological Features of Drug-Induced Liver Injury
}

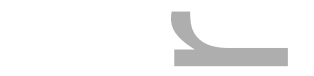 \\ Ayako Suzuki ${ }^{1}$, Huiman Barnhart ${ }^{2}$, Jiezhun $\mathrm{Gu}^{2}$, Herbert L. Bonkovsky ${ }^{3,4}$, Hans L. Tillmann ${ }^{5}$, \\ Robert J. Fontana ${ }^{6}$, David E. Kleiner ${ }^{7}$, for Drug-induced Liver Injury Network (DILIN) \\ ${ }^{1}$ Gastroenterology, Duke University, Durham, NC \\ 2Duke Clinical Research Institute, Durham, NC \\ ${ }^{3}$ Section on Gastroenterology \& Hepatology, Wake Forest University School of Medicine, \\ Winston-Salem, NC \\ ${ }^{4}$ University of North Carolina, Chapel Hill, NC \\ ${ }^{5}$ Gastroenterology, Brody School of Medicine, East Carolina University, Greenville, NC
}

This is the author manuscript accepted for publication and has undergone full peer review but has not been through the copyediting, typesetting, pagination and proofreading process, which may lead to differences between this version and the Version of Record. Please cite this article as doi: $\underline{10.1111 / \text { liv.13380 }}$

This article is protected by copyright. All rights reserved 
3

4 Corresponding Author:

5 Ayako Suzuki, MD, PhD, MSc

6

7

8

9

10

11

12

13

14

15

Gastroenterology, Duke University

200 Trent Dr. DUMC3913

Durham, NC 27710

Email: ayako.suzuki@duke.edu

Abstract: 250 words

Words: 4891 words

Figures and Tables: 3 Tables confidence interval. Cancer Institute.

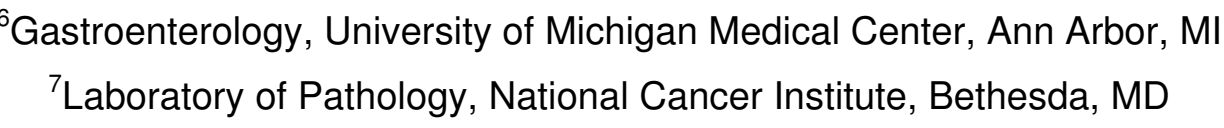

${ }^{7}$ Laboratory of Pathology, National Cancer Institute, Bethesda, MD

TEL: 919-684-6211/FAX: 919-681-8147

Abbreviations: DILI: drug-induced liver injury; DILIN: drug-induced liver injury network; HC: hepatocellular; CS/MIX: cholestatic/mixed; ALT: alanine aminotransferase; ALP: alkaline phosphatase; ULN: upper limit normal; ANA: antinuclear antibody; OR: odds ratio; CI:

Financial support: The DILIN is structured as an U01 cooperative agreement supported by the National Institute of Diabetes and Digestive and Kidney Diseases (NIDDK) and the National Institutes of Health (NIH) with funds provided by the following grants: U01DK065211 (Indiana University [Purdue]), U01DK065184 (University of Michigan [Ann Arbor]), U01DK065201 (University of North Carolina [Chapel Hill, Asheville, Wake Forest \{Wake Forest Baptist Medical Center]), U01DK083020 (University of Southern California, University of California-Los Angeles [Pfleger Liver Institute]), U01DK083027 (Albert Einstein Medical Center), U01DK100928 (Icahn 7 School of Medicine at Mount Sinai), U01DK065176 (Duke Clinical Research Institute). This 8 research was also supported in part by the Intramural Research Program of the $\mathrm{NIH}$, National

This article is protected by copyright. All rights reserved 
1 Conflict of Interest: The authors have the following disclosures: Ayako Suzuki: served as a

2 consultant for GlaxoSmithKline; Herbert L. Bonkovsky: served as a consultant to Alnylam,

3 Clinuvel, Mitsubishi-Tanabe, Moderna, Recordati, and Stoke Pharma in the past 12 months,

4 research support from Alnylam, Clinuvel, and Gilead Sciences ; Robert J. Fontana: grant

5 support from Gilead, BMS, Jansen and Vertex and served as a paid consultant to Tibotec,

6 Merck and GlaxoSmithKline; Drs Gu, Barnhart, Tillmann, and Kleiner have no financial

7 disclosures relating to this work.

\section{Key points}

- Liver biopsies obtained within 30 days of onset from 212 patients with drug-induced liver injury were analyzed for the associations of gender and a proxy of female menopausal status with histologic features in the acute injury phase.

- Biopsies of the 58 women $<50$ years of age were associated with more severe interface

4
hepatitis and less iron-stained hepatocytes vs. those of the 87 men and 67 women $\geq 50$ years.

- Compared to those of men, biopsies of women showed significantly greater plasma cell infiltration, hepatocyte apoptosis, hepatocyte rosettes, and lobular disarray.

- Biopsies from men showed greater histological cholestasis.

\section{Abstract}

[Background and aim] Gender and menopause may contribute to type and severity of druginduced liver injury (DILI) by influencing host responses to injury. The aim of this study was to assess the associations of gender and female age 50 [a proxy of menopause] with histologic features of liver injury in 212 adults enrolled in the Drug-Induced Liver Injury Network (DILIN) registry. [Methods] All participants had a causality score of at least 'probable', a liver biopsy within 30 days of DILI onset, and no prior chronic liver disease. Biochemical and histologic injury types were classified as hepatocellular or cholestatic/mixed injury. The cohort was divided into three gender/age categories: men $(41.0 \%)$, women $<50$ years $(27.4 \%)$, and women $\geq 50$ years of age (31.6\%). Interaction of gender and age category ( $\geq 50$ or not) was assessed. [Results] Hepatocellular injury was more prevalent in women $<50$ years vs. others $(p=0.002)$. After adjusting for biochemical injury types, black race and possible aging effects, more severe interface hepatitis was noted in biopsies of women $<50$ years compared to those of men and women $\geq 50$ years ( $p=0.009$ and $p=0.055$, respectively). Compared to those of men, biopsies of women showed greater plasma cell infiltration, hepatocyte apoptosis, hepatocyte rosettes, and 
lobular disarray but less iron-positive hepatocytes and histological cholestasis $(p<0.05)$. These associations persisted after excluding cases of amoxicillin/clavulanic acid, anabolic steroids or nitrofurantoin DILI which showed gender-specific distributions. [Conclusion] Gender and a proxy of menopause were associated with various features of inflammation and injury in DILI.

Key words: drug-induced liver injury, gender difference, menopause, liver histology,

hepatotoxicity. \\ 8 INTRODUCTION}

Significant heterogeneity exists in clinical phenotypes of drug-induced liver injury (DILI). Initial biochemical presentations, autoimmune features, liver histology, and clinical outcomes vary substantially among individuals who develop DILI, even when caused by the same agent.[1-4]

12 Such heterogeneity suggests that how individuals respond to drug toxicity, cellular stress and tissue injury may contribute to the variable clinical phenotypes of DILI.

Gender and sex hormones influence drug metabolism and transport.[5, 6] They also modulate host responses to injury; sexual dimorphism in cellular stress response, cell death, immune response, inflammation and tissue repair has been demonstrated in various systems. In particular, sexual dimorphism in the immune response has been well documented in humans as well as experimental models.[7] Several recent experimental studies demonstrated sex differences in host responses to injury. An immune-mediated DILI model showed sex differences in immune response and inflammation: more severe hepatitis, more antibody production, and a higher level of pro-inflammatory hepatic cytokines in females vs. male mice.[8] In the halothane-induced liver injury mouse model, estrogens reduce injury while progesterone exacerbates injury.[9, 10] In an immune-mediated nephritis model more apoptosis occurred in females vs. more necrosis in males, and the administration of estrogen to male mice induced apoptosis and inhibited necrosis.[11] Taken together, these findings suggest that gender and sex hormones may modulate host responses to liver injury insults and contribute to diverse clinical manifestation and severity of human DILI.

We hypothesized that gender and sex hormones modulate cellular stress responses, cell death, immune responses, and inflammation in drug toxicity and may influence clinical and histologic DILI manifestations in humans. We aimed to investigate the associations of gender and women's age (with $\geq 50$ years as a surrogate for menopause) with histologic features in acute phase of DILI, for the purpose of further refining the above-mentioned hypothesis. Our thorough 
descriptive analyses using different modeling approaches identified several histologic features associated with gender (and women's age < 50) across initial biochemical presentations even after excluding gender-specific causal agents. The findings support the above-mentioned hypothesis and also pose important clinical and pathophysiological questions relevant to gender-differences in DILI phenotypes, which should be further investigated across different disciplines.

\section{METHODS}

\section{Study design and data source}

This is a hypothesis-driven, cross sectional analysis designed to investigate the associations of gender and female menopausal status with various histologic features in patients with DILI.

Data from the U.S. Drug-Induced Liver Injury Network (DILIN) were utilized in our analysis. The study design and data collection in the prospective DILIN database study have been previously described.[12] Briefly, the consortium enrolls consecutive adult and pediatric patients with suspected DILI. Detailed clinical information, including laboratory data, medications, medical \& social history, and symptoms and signs at DILI onset, was collected at the time of study enrollment. DILI onset was defined per protocol as date of initial presentation with elevated liver enzymes that met any of the study criteria.[12] Causality assessment was performed by the DILIN causality committee in a consensus manner using the DILIN causality score after 6 months follow-up. For subjects who showed evidence of persisting DILI [13] , additional followup evaluations were performed at 12 months and 24 months. [12] Liver biopsy was not required for study enrollment. When liver biopsy was performed for clinical indications, biopsy slides were obtained from the clinical study centers. The clinical decision on whether to perform a liver biopsy was solely made by the attending physician who often differed from the DILIN investigator. The DILIN studies were approved by the Institutional Review Board (IRB) at each participating center (listed in Supplemental Methods). Informed consent was obtained from each of the participants prior to the study enrollment.

\section{Study population}

Of 1386 enrolled patients between September 2004 and May 2014, 212 (15\%) patients who met the following criteria were analyzed: 1) Age > 18 years 2) an adequate, evaluable liver biopsy that had been performed within 30 days of DILI onset, 3) a DILIN causality score of 'probable' or 
1 higher [14], 4) no prior diagnosis of other chronic liver diseases. The time window for this study

2 was set to focus on histologic features in acute phase.

\section{Study variables}

Predictors: The primary predictor variable tested in this study was the gender/female menopause classification. As reproductive information was not collected as a part of the DILIN study, age 50 years old, the average age of female menopause in the US, was used as a surrogate.[15] Prior to our main analyses, we performed background analyses to assess an interaction between gender and age 50 in each histologic feature to better characterize aging effect vs. menopausal effect as detailed in Supplemental Statistical Method. Based on the background analyses, the study population was classified into 3 categories (hereafter called the gender/age categories), men, women younger than 50 years (surrogate for premenopausal), and women 50 years old or older (surrogate for postmenopausal) in our main analyses. Gender (men vs. all women) was also assessed as the secondary predictor.

Outcomes: Liver biopsy slides stained with hematoxylin-eosin and Masson trichrome stain were obtained from the clinical study centers and were reviewed and scored in a blinded, standardized manner by a single experienced hepatopathologist [DEK].[16] Histologic features, including interface hepatitis, plasma cell infiltration, cholestatic degree, hepatocellular cholestasis, canalicular cholestasis, hepatocyte rosettes, lobular disarray, iron stainhepatocellular iron, and apoptosis, were analyzed as primary study outcomes in this study. Histologic injury types were classified into the following two categories based on the histologic injury patterns: cholestatic/mixed injury (i.e., acute cholestatic, chronic cholestatic, and combined hepatitic/cholestatic) and hepatocellular (i.e., others).[16]

Others: Initial biochemical injury types, hepatocellular $[\mathrm{HC}]$ injury and cholestatic/mixed [CS/MIX] injury, were determined based on the $\mathrm{R}$ ratio at the time of DILI onset or the closest to the onset when the laboratory data were not available at the onset, calculating the ratio of serum alanine aminotransferase (ALT)/upper limit normal (ULN) to serum alkaline phosphatase $(\mathrm{ALP}) / \mathrm{ULN}:>=5(\mathrm{HC})$ vs. $<5$ (CS/MIX).[17] R-values were calculated using institutional reference ranges of serum ALT and ALP at the time of case enrollment. Information on demography, laboratory data at the study enrollment, and suspected drugs was also obtained for our analyses. 


\section{Statistical analyses}

Descriptive data are reported as mean \pm standard deviation or median with interquartile range for continuous variables and as a percentage for categorical variables. To assess selection bias due to the lack of liver biopsy within 30 days of DILI onset, clinical characteristics of the study population were compared between those included in the analysis versus those not included in the analysis (i.e., age > 18 years, a DILIN causality score of 'probable' or higher, and no prior diagnosis of other chronic liver diseases, but no biopsies within 30 days of DILI onset).

Clinical characteristics of the study population were compared among the gender/age categories by using Kruskal-Wallis test for continuous and ordinal variables and chi-square test for categorical variables. Histological features of the study population were similarly compared among the gender/age categories with stratification on different biochemical injury types.

For modeling the histologic features, univariate analysis was performed first to select potential clinical covariates for adjustment in the multivariate models. To determine a proper age variable to be included in a model, background analyses were performed as described in Supplemental Statistical Methods. Depending on the histologic outcomes (binary vs. ordinal), logistic regression models or ordinal logistic regression models were used. For the ordinal logistic regression, proportional odds models or cumulative logistic regression models were selected, depending on whether or not the proportional odds assumption was met for a specific histologic outcome. Adjusted odds ratio or cumulative odds ratio with $95 \%$ confidence interval $(\mathrm{Cl})$ and $\mathrm{p}$ value were reported. The fit of the models was assessed with Hosmer-Lemeshow goodness-offit test for binary and ordinary outcomes.[18]

Statistical analyses were performed using SAS version 9.4. (SAS Institute, Cary, NC). All $P$ values presented are 2-sided, and the differences were considered statistically significant when the $\mathrm{P}<0.05$. For detecting interactions in the background analyses, we used $\mathrm{p}<0.1$ due to the small sample size. Because of the descriptive nature of this analysis, $P$ values have not been adjusted for multiple comparisons.

\section{RESULTS}

\section{Clinical characteristics}


A total of 212 adults with DILI were included in the analysis. Clinical characteristics of the study population at enrollment are summarized in Table 1 . Women of age $<50$ years, women of age $\geq 50$ years, and men comprised $27.4 \%, 31.6 \%$, and $41.0 \%$ of the study population, respectively.

4 Mean $[ \pm S D]$ age of the total study population was $50 \pm 16$ years old. Seventy-seven percent were white, $14.6 \%$ black, and 10\% Hispanic. Fifty percent of the cases presented as HC injury at DILI onset. Age, the biochemical presentation at DILI onset, serum ALT, serum AST, serum ALP, and positive ANA were significantly different among the gender/age categories (Table 1). Hepatocellular injury was noted in $70.7 \%$ of women of $<50$ years compared to $43.3 \%$ of women of $>=50$ years and $41.4 \%$ of men. Serum ALT and AST were the highest in women aged $<50$ years, while serum ALP was the highest in women aged $\geq 50$ years. Men had a lower frequency of positive ANA (14.5\%) compared to women ( $>30 \%$ in both groups) at the study enrollment. Black, other race, and Hispanic were more prevalent among women of age $<50$ years although there were no statistical significances.

The clinical characteristics of the study population were then compared with the patients excluded from this analysis due to the lack of liver biopsy within 30 days of the DILI onset. The clinical characteristics of the study population $(\mathrm{N}=212) \mathrm{vs}$. the population not included in this analysis ( $\mathrm{N}=792)$ are summarized in Supplementary Table 2. Briefly, clinical severity score, serum ALP, and total bilirubin at DILI onset were higher in the study population. The age, the gender/age categories, self-reported race/ethnicity, biochemical injury type at DILI onset, and positive ANA did not show statistical differences between the populations. Biochemical injury type, clinical severity score, and positive ANA were further analyzed, classifying by the age/gender categories in each population (Supplementary Table 3). No statistically significant interactions (the populations $x$ the age/gender categories) were noted.

Causal agents implicated in the study population are provided in Supplementary Table 1a and 1b. Among agents implicated in $\geq 4$ cases (Table 1), Herbs-Dietary Supplement (HDS) was most prevalent (25\%), followed by amoxicillin/clavulanic acid (12.2\%). Eleven cases of the HDSrelated DILI were caused by anabolic steroids, all of whom were males.

\section{Univariate associations of the histologic features with the gender/age categories}

The previous analysis demonstrated the histologic features significantly differ depending on the initial biochemical presentations: hepatocellular vs. cholestatic/mixed injury.[16] Therefore, the univariate associations were assessed not only in the total study population but also within the groups of hepatocellular injury and cholestatic/mixed injury separately (Table 2). Several 
variables were significantly associated with the gender/age categories. It is notable that, across the injury types, interface hepatitis, noticeable increase in plasma cells, apoptosis, hepatocyte rosettes, and lobular disarray showed a female-dominant pattern while cholestasis and hepatocyte iron-positivity by Perls' staining showed a male-dominant pattern. These histologic

5 features were considered in the multivariable analyses.

\section{Adjusted associations of the histologic features with the gender/age categories}

In our background analyses, a potential effect of advancing age was only evident in interface hepatitis at age of 70 in both men and women. The age category ( $<50 \mathrm{vs}$. $\geq 50)$ did not show significant associations with any histologic features in men. There was an interaction of gender and the age category in the histologic features of interface hepatitis $(P=0.05)$ and apoptosis $11(p=0.06)$.

The adjusted associations of histologic features with 1) the gender/age categories (Model 1) and 2) gender (Model 2) are presented in Table 3. All the models were adjusted for biochemical injury types, black race, and, for interface hepatitis only, the age category (<70 vs. $\geq 70$ ). The goodness of fit tests indicated an adequate fit ( $p$-values of 0.27 to 0.99 ). More severe interface hepatitis and less hepatocyte iron stains were noted in biopsies of women < 50 years compared to those of men and women $\geq 50$ years $(p=0.009$ and $p=0.055$ for interface hepatitis and $p<0.001$ and $p=0.046$ for hepatocyte iron stains, respectively). Compared to those of men, biopsies of women were associated with more plasma cell infiltration, more apoptosis, more hepatocyte rosettes, more lobular disarray, and less iron-stained hepatocytes and less cholestasis $(\mathrm{p}<0.05)$.

Amoxicillin/clavulanic acid, anabolic steroids and nitrofurantoin, which showed gender-specific distributions in this study population, have been associated with signature injury patterns. Therefore, we assessed the associations between the histologic features and the gender/age categories (or gender) after excluding these agents. These associations remained similar even after excluding cases due to anabolic steroids, nitrofurantoin, or amoxicillin/clavulanic acid (data are not shown).

The associations of histologic features with the gender/age categories and gender were also assessed using the same modeling strategy but adjusting for the histologic injury classification, 
of men vs. women were decreased for hepatocellular/canalicular cholestasis (data are not

2 shown).

\section{DISCUSSION}

This hypothesis-refining analysis revealed intriguing findings pertaining to gender-specific phenotypes of DILI. Women $<50$ years of age were more likely to show severe interface hepatitis and less hepatocyte iron staining compared to men and women $\geq 50$ years. The comparable risk reduction in women $\geq 50$ years and men vs. women $<50$ years of age implies the involvement of female sex hormones as opposed to innate sex difference in the pathology involved in these features. For this interpretation, we considered a possible indication bias; young women with positive ANA and high serum ALT and AST might have undergone liver biopsies more often than others to rule out autoimmune hepatitis. However, we found no evidence of bias regarding frequencies of liver biopsies that could explain more severe autoimmune hepatitis features in women aged $<50$ vs. women aged $\geq 50$ and men (Supplementary Tables 2 and 3); the study population appeared to have similar proportions of positive ANA in each age/gender category as compared to the excluded population (interaction $P=0.29$ ). Positive ANA is observed among healthy general population.[19, 20] Thus whether positive ANA is an innocent bystander, or a risk factor of developing hepatotoxicity, or a consequence of hepatotoxicity observed in a subgroup of patients is uncertain. Numbers of ironpositive hepatocytes were the highest in men, intermediate in women $\geq 50$ years, and the lowest in women $<50$ years of age. Similar male dominance in hepatic iron deposition has been reported in a cohort of NAFLD.[21] This might be explained by blood loss of menstruation, pregnancy, and breast feeding and/or estrogen's effect on hepcidin.[22]

Men had a higher likelihood of cholestatic features with a higher severity compared to women. Of note, our 'risk of bias' assessment showed comparable biochemical injury type within the age/gender categories between the study population and the excluded population (interaction $\mathrm{P}=0.67$ in Supplemental Table 3). The consistent observation after excluding the cases caused by anabolic steroids or amoxicillin/clavulanic acid ruled out the possible explanation by male dominance observed with these agents (data not shown). Unlike the autoimmune hepatitis features, the gender difference in cholestatic features appeared to be consistent before and after the age 50 years. Our extended analysis showed that male gender (OR and $95 \% \mathrm{Cl}=2.0$ $[1.1,3.6], p=0.019$ ) and age $\geq 50$ years (OR and 95\% $\mathrm{Cl}=3.0[1.7,5.4], p=0.0001$ ) were independently associated with the cholestatic/mixed injury $(R \leq 2)$. This observation is consistent 
with previous observational studies.[3, 23] Although underlying mechanisms are uncertain,

2 these findings suggest a gender difference in cholestasis, independent of age and female sex

3 hormones. The models adjusting for the histologic injury classification showed consistent

4 associations except for hepatocellular/canalicular cholestasis. Hepatocellular/canalicular

5 cholestasis could develop in severe hepatocellular injury as a consequence of compromised

6 cellular energy supply and subsequent impairment of highly energy-dependent transporters

7 such as BSEP [24], which are difficult to distinguish from true cholestatic injury without

8 considering overall histologic pictures.

9 It is well known that women are more prone to develop autoimmune disorders of all types than are men and that sex hormones significantly modulate innate as well as adaptive immune responses.[7] Women in general induce stronger antibody-production and cell-mediated

12 immune responses following either infection or vaccination than men.[7] Also, there are quite a

13 few experimental studies showing how estrogens and progesterone influence cellular stress

14 response and severity of liver injury. Estrogens are in general reported to be protective against

15 liver injury $[25,26]$ while progesterone appears to exert detrimental impacts on inflammation

16 and fibrosis.[27, 28] Thus, impact of sex and sex hormones on host response to drug toxicity is

17 likely multilayered [29] and modulates gender-specific clinical phenotypes.

Another noteworthy finding in our analysis was that black race was associated with about 2-fold higher likelihood of having more noticeable plasma cell infiltration across the different models ( $p$-value of 0.048 to 0.08 ; data are not shown). This is intriguing as black race is reportedly associated with an enhanced humoral immunological response following vaccination compared to other races.[30] A previous study reported that black women were overrepresented among non-acetaminophen induced acute liver failure.[31, 32] As women were associated with more noticeable plasma cell infiltration, there may be an additive interaction between gender and genetic predisposition causing an enhanced humoral immune response in DILI susceptibility and DILI severity.

A strength of this study is that the analysis was performed using a well-characterized DILI population with careful follow-up for up to two years in which data were collected and evaluated in a standardized manner.[14, 33] Also, we applied different modeling approaches to enhance our data interpretation and theory-generation. This study also has several limitations. We used a

32 proxy of menopause and the information on exact menopausal state was not available. Possible misclassification among women may have blunted the true associations. Sex hormone levels 
were not obtained. Our study population may have been biased due to the requirement of liver biopsy data. Although we evaluated the 'risk of bias' and discussed it above, there may be a bias unmeasured in this study. Therefore, our findings may be applicable only to those who developed clinically significant drug-induced liver injury and who are likely considered for liver biopsy. The initiation of liver injury and the type of initial presentation are likely a function of drug and host and may be determined by their specific interplay.[29] Host-drug interplay in DILI is beyond the scope of this analysis. Our analysis was meant to address the impact of gender and menopause on 'host response' to DILI 'after' the initiation of injury, but not impact on the initiation of liver injury or tissue recovery. Host response to drug toxicity is likely modified by multiple host factors, including concomitant medications and pre-existing co-morbidities,[29] which were not analyzed in this analysis. Race/ethnicity, other than Caucasian or black, also could not be included in this analysis due to their low frequencies. Lastly, our analytic strategy was built based on our hypothesis. Whether our analytic approach is justifiable from a mechanistic viewpoint may be questioned. We separately developed a model considering all the available variables at study enrollment, independent of the hypothesis. This model also identified gender as a significant contributor in the histologic features analyzed in this study, which further supports the significance of gender in DILI histology.

In summary, our analysis demonstrated that histologic features of DILI were significantly associated with gender and age [used as a proxy of menopause] in patients presenting with varying laboratory profiles. The findings support our hypothesis and generated several additional intriguing hypotheses relevant to gender differences in injury/stress responses to drug toxicity (e.g., cell death pathway, inflammation, and immune response). Further clinical analyses and the translation to experimental studies are warranted to delineate gender-dependent hepatotoxicity and DILI manifestations.

\section{REFERENCES}

1. Uetrecht, J., Immunoallergic drug-induced liver injury in humans. Semin Liver Dis, 2009. 29(4): p. 383-92. Lucena, M.I., R.J. Andrade, M.C. Fernández, et al., Determinants of the clinical expression of amoxicillin-clavulanate hepatotoxicity: A prospective series from Spain. Hepatology, 2006. 44(4): p. $850-856$.

3. Lucena, M.I., R.J. Andrade, N. Kaplowitz, et al., Phenotypic characterization of idiosyncratic druginduced liver injury: The influence of age and gender. Hepatology, 2009 49(6): p. 2001-2009.

This article is protected by copyright. All rights reserved 
1 4. Liu, Z.X. and N. Kaplowitz, Immune-mediated drug-induced liver disease. Clin Liver Dis, 2002. 2 6(3): p. 755-74.

35 5ang, L., Y. Li, H. Hong, et al. Sex Differences in the Expression of Drug-Metabolizing and

9. Toyoda, Y., S. Endo, K. Tsuneyama, et al., Mechanism of exacerbative effect of progesterone on

15. Nichols, H.B., A. Trentham-Dietz, J.M. Hampton, et al., From menarche to menopause: trends

17. Benichou, C., Criteria of drug-induced liver disorders. Report of an international consensus

10. Toyoda, Y., T. Miyashita, S. Endo, et al., Estradiol and progesterone modulate halothane-induced liver injury in mice. Toxicol Lett, 2011. 204(1): p. 17-24.

11. Jog, N.R. and R. Caricchio, Differential regulation of cell death programs in males and females by Poly (ADP-Ribose) Polymerase-1 and 17beta estradiol. Cell Death Dis, 2013. 4: p. e758.

12. Fontana, R.J., P.B. Watkins, H.L. Bonkovsky, et al., Drug-Induced Liver Injury Network (DILIN) prospective study: rationale, design and conduct. Drug Saf, 2009. 32(1): p. 55-68.

13. Fontana, R.J., P.H. Hayashi, H. Barnhart, et al., Persistent liver biochemistry abnormalities are more common in older patients and those with cholestatic drug induced liver injury. Am J Gastroenterol, 2015. 110(10): p. 1450-9.

14. Chalasani, N., R.J. Fontana, H.L. Bonkovsky, et al., Causes, clinical features, and outcomes from a prospective study of drug-induced liver injury in the United States. Gastroenterology, 2008. 135(6): p. 1924-34, 1934 e1-4. among US Women born from 1912 to 1969. Am J Epidemiol, 2006. 164(10): p. 1003-11.

16. Kleiner, D.E., N.P. Chalasani, W.M. Lee, et al., Hepatic histological findings in suspected druginduced liver injury: systematic evaluation and clinical associations. Hepatology, 2014. 59(2): p. 661-70. meeting. J Hepatol, 1990. 11(2): p. 272-6. 
1 18. Fagerland, M.W. and D.W. Hosmer, A goodness-of-fit test for the proportional odds regression 2 model. Stat Med, 2013. 32(13): p. 2235-49.

3 19. Teubner, A., H.L. Tillmann, D. Schuppan, et al., [Prevalence of circulating autoantibodies in

22. Ikeda, Y., S. Tajima, Y. Izawa-Ishizawa, et al., Estrogen regulates hepcidin expression via GPR30-

23. De Valle, M.B., V. Av Klinteberg, N. Alem, R. Olsson, and E. Bjornsson, Drug-induced liver injury in

24. Shiba, Y. and Y. Kanno, Effects of ATP depletion with DL-ethionine on biliary excretion of

25. Xu, J.W., J. Gong, X.M. Chang, et al., Estrogen reduces CCL4- induced liver fibrosis in rats. World J

26. Zhang, Y., L. Wu, Y. Wang, et al., Protective role of estrogen-induced miRNA-29 expression in carbon tetrachloride-induced mouse liver injury. J Biol Chem, 2012. 287(18): p. 14851-62.

29. Chen, M., A. Suzuki, J. Borlak, R.J. Andrade, and M. Isabel Lucena, Drug-Induced liver injury:

30. Haralambieva, I.H., H.M. Salk, N.D. Lambert, et al., Associations between race, sex and immune response variations to rubella vaccination in two independent cohorts. Vaccine, 2014. 32(17): p. 1946-53. 
1 31. Russo, M.W., J.A. Galanko, R. Shrestha, M.W. Fried, and P. Watkins, Liver transplantation for acute liver failure from drug induced liver injury in the United States. Liver Transpl, 2004. 10(8):

3 p. 1018-23.

$4 \quad 32 . \quad$ Reuben, A., D.G. Koch, W.M. Lee, and Acute Liver Failure Study Group, Drug-induced acute liver failure: results of a U.S. multicenter, prospective study. Hepatology, 2010. 52(6): p. 2065-76.

6 33. Chalasani, N., H.L. Bonkovsky, R. Fontana, et al., Features and Outcomes of 899 Patients With 7 Drug-Induced Liver Injury: The DILIN Prospective Study. Gastroenterology, 2015. 148(7): p. 134052 e7.
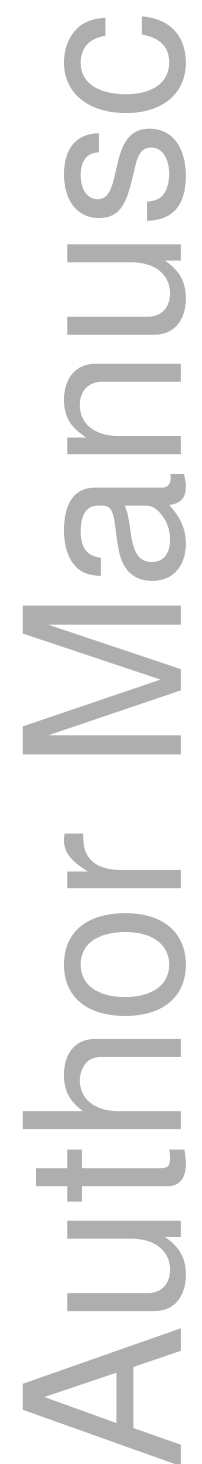
Table 1: Clinical Characteristics of the study population

\begin{tabular}{|c|c|c|c|c|c|}
\hline \multirow{2}{*}{ Clinical characteristics } & Total & Women $<50$ & Women $>=50$ & Men & \multirow{2}{*}{$p$-value } \\
\hline & $\mathrm{N}=212$ & $N=58$ & $N=67$ & $N=87$ & \\
\hline Age, year, mean $\pm S D$ & $50.1 \pm 15.7$ & $37.1 \pm 9.3$ & $62.4 \pm 9.0$ & $49.4 \pm 15.9$ & $<0.001$ \\
\hline Race & & & & & 0.077 \\
\hline White, \% & 77.4 & 63.8 & 80.6 & 83.9 & \\
\hline Black, \% & 14.6 & 24.1 & 11.9 & 10.3 & \\
\hline Others, $\%$ & 8.0 & 12.1 & 7.5 & 5.7 & \\
\hline Ethnicity Hispanic, \% & 10.0 & 15.5 & 9.1 & 6.9 & 0.242 \\
\hline Biochemical injury type: Hepatocellular injury*, \% & 50.0 & 70.7 & 43.3 & 41.4 & 0.002 \\
\hline \multicolumn{6}{|l|}{ Liver chemistries at onset, median $\left(25^{\text {th }}, 75^{\text {th }}\right)$} \\
\hline $\mathrm{ALT}, \mathrm{U} / \mathrm{L} \bigcirc$ & $538(245,1268)$ & $770(267,1522)$ & $649(331,1193)$ & $418(173,948)$ & 0.011 \\
\hline AST, U/L & $353(137,989)$ & $738(224,1437)$ & $557(162,1092)$ & $205(86,553)$ & $<0.001$ \\
\hline$A L P, U / L$ & $245(161,385)$ & $215(153,306)$ & $315(220,426)$ & $225(138,387)$ & $<0.001$ \\
\hline Total bilirubin, $\mathrm{mg} / \mathrm{dL}$ & $7.3(3.8,11.9)$ & $5.8(3.3,10.4)$ & $6.6(3.4,11.9)$ & $8.5(5.0,12.9)$ & 0.054 \\
\hline INR & $1.2(1.0,1.5)$ & $1.2(1.1,1.6)$ & $1.2(1.0,1.7)$ & $1.1(1.0,1.3)$ & 0.336 \\
\hline Positive ANA, $\%$ & 26.7 & 30.4 & 38.8 & 14.5 & 0.002 \\
\hline Time from DILI onset to Liver biopsy, days & $9[5,15]$ & $7[5,13]$ & $9[5,15]$ & $9[4,15]$ & 0.4671 \\
\hline \multicolumn{6}{|l|}{ Primary implicated agents, $\mathrm{N}^{* *}$} \\
\hline Herbs-Dietary Supplements ${ }^{\#}$ & 53 & 10 & 11 & 32 & \\
\hline Amoxicillin/clavulanic acid & 26 & 2 & 7 & 17 & \\
\hline Minocycline & 8 & 4 & 2 & 2 & \\
\hline Nitrofurantoin & 6 & 1 & 5 & 0 & \\
\hline Cefazolin & 6 & 2 & 3 & 1 & \\
\hline
\end{tabular}

This article is protected by copyright. All rights reserved 


\begin{tabular}{|l|c|c|c|}
\hline Ciprofloxacin & 5 & 1 & 3 \\
\hline Levofloxacin & 4 & 1 & 1 \\
\hline Allopurinol & 4 & 0 & 2 \\
\hline
\end{tabular}

*: R-value $>=5 . * *$ : Distributions of implicated drugs $(>=4)$ are provided above. \#: Of them, eleven cases were caused by anabolic steroids.

Table 2: Univariate associations of the gender/age group categories with histologic features

\begin{tabular}{|c|c|c|c|c|c|c|c|c|c|c|c|c|}
\hline & \multicolumn{4}{|c|}{ Total population } & \multicolumn{4}{|c|}{ Hepatocellular injury } & \multicolumn{4}{|c|}{ Cholestatic/mixed injury } \\
\hline & $F<50$ & $F>=50$ & Men & & $\mathrm{F}<50$ & $F>=50$ & Men & & $F<50$ & $F>=50$ & Men & \\
\hline & $N=58$ & $N=67$ & $\mathrm{~N}=87$ & & $\mathrm{~N}=41$ & $\mathrm{~N}=29$ & $\mathrm{~N}=36$ & & $\mathrm{~N}=17$ & $\mathrm{~N}=38$ & $N=51$ & \\
\hline Interface Hepatitis, \% & & & & $* *$ & & & & $*$ & & & & \\
\hline Grade 0 & 3.6 & 4.5 & 10.6 & & 0.0 & 0.0 & 11.8 & & 11.8 & 8.1 & 9.8 & \\
\hline Grade 1 & 19.6 & 40.9 & 35.3 & & 12.8 & 20.7 & 20.6 & & 35.3 & 56.8 & 45.1 & \\
\hline Grade 2 & 10.7 & 16.7 & 25.9 & & 10.3 & 13.8 & 26.5 & & 11.8 & 18.9 & 25.5 & \\
\hline Grade 3 & 26.8 & 13.6 & 14.1 & & 30.8 & 17.2 & 11.8 & & 17.6 & 10.8 & 15.7 & \\
\hline Grade 4 & 39.3 & 24.2 & 14.1 & & 46.2 & 48.3 & 29.4 & & 23.5 & 5.4 & 3.9 & \\
\hline Noticeable plasma cell infiltration, \% & 37.5 & 31.8 & 11.6 & $* *$ & 41.0 & 53.6 & 14.3 & $*$ & 29.4 & 15.8 & 9.8 & \\
\hline Cholestasis, degree, \% & & & & $* *$ & & & & $*$ & & & & $* *$ \\
\hline Grade 0 & 48.2 & 46.3 & 19.8 & & 53.8 & 58.6 & 31.4 & & 35.3 & 36.8 & 11.8 & \\
\hline Grade 1 & 17.9 & 16.4 & 14.0 & & 23.1 & 24.1 & 22.9 & & 5.9 & 10.5 & 7.8 & \\
\hline Grade 2 & 26.8 & 20.9 & 27.9 & & 17.9 & 10.3 & 34.3 & & 47.1 & 28.9 & 23.5 & \\
\hline Grade 3 & 7.1 & 16.4 & 38.4 & & 5.1 & 6.9 & 11.4 & & 11.8 & 23.7 & 56.9 & \\
\hline Hepatocellular cholestasis, \% & 41.1 & 47.8 & 72.1 & $* *$ & 30.8 & 31.0 & 57.1 & $*$ & 64.7 & 60.5 & 82.4 & \\
\hline Canalicular cholestasis, \% & 50.0 & 52.2 & 74.4 & $* *$ & 43.6 & 41.4 & 62.9 & & 64.7 & 60.5 & 82.4 & \\
\hline Hepatocyte rosettes (more than rare), \% & 51.8 & 30.3 & 17.6 & $* *$ & 61.5 & 53.6 & 38.2 & & 29.4 & 13.2 & 3.9 & $*$ \\
\hline
\end{tabular}

This article is protected by copyright. All rights reserved 


\begin{tabular}{|c|c|c|c|c|c|c|c|c|c|c|c|c|}
\hline Lobular disarray present, \% & 42.9 & 24.2 & 15.1 & $* *$ & 53.8 & 46.4 & 34.3 & & 17.6 & 7.9 & 2.0 & $*$ \\
\hline Iron-stain-hepatocytes, \% & & & & $* *$ & & & & $*$ & & & & $* *$ \\
\hline Grade 0 & 81.5 & 63.9 & 35.9 & & 81.6 & 65.4 & 46.7 & & 81.3 & 62.9 & 29.2 & \\
\hline Grade $1-2$ & 18.5 & 36.1 & 64.1 & & 18.4 & 34.6 & 53.3 & & 18.8 & 37.1 & 70.8 & \\
\hline Apoptosis, \% & & & & $* *$ & & & & & & & & \\
\hline Grade 0 & 10.7 & 17.9 & 22.4 & & 5.1 & 6.9 & 11.8 & & 23.5 & 26.3 & 29.4 & \\
\hline Grade 1 & 33.9 & 47.8 & 61.2 & & 28.2 & 31.0 & 47.1 & & 47.1 & 60.5 & 70.6 & \\
\hline Grade 2 & 55.4 & 34.3 & 16.5 & & 66.7 & 62.1 & 41.2 & & 29.4 & 13.2 & 0.0 & \\
\hline Lobular inflammation, \% & & & & & & & & $*$ & & & & \\
\hline Grade 0 & 0.0 & 0.0 & 1.2 & & 0.0 & 0.0 & 2.9 & & & & & \\
\hline Grade 1 & 10.7 & 10.4 & 11.6 & & 2.6 & 10.3 & 11.4 & & 29.4 & 10.5 & 11.8 & \\
\hline Grade 2 & 5.4 & 17.9 & 17.4 & & 2.6 & 10.3 & 11.4 & & 11.8 & 23.7 & 21.6 & \\
\hline Grade 3 & 17.9 & 25.4 & 23.3 & & 12.8 & 6.9 & 17.1 & & 29.4 & 39.5 & 27.5 & \\
\hline Grade 4 & 66.1 & 46.3 & 46.5 & & 82.1 & 72.4 & 57.1 & & 29.4 & 26.3 & 39.2 & \\
\hline Lymphoid aggregates/Germinal centers, \% & 3.6 & 12.1 & 4.7 & & 2.6 & 25.0 & 8.6 & $*$ & 5.9 & 2.6 & 2.0 & \\
\hline
\end{tabular}

\begin{tabular}{|c|c|c|c|c|c|c|c|c|c|c|c|c|}
\hline & \multicolumn{4}{|c|}{ Total population } & \multicolumn{4}{|c|}{ Hepatocellular injury } & \multicolumn{4}{|c|}{ Cholestatic/mixed injury } \\
\hline & $\mathrm{F}<50$ & $F>=50$ & Men & & $\mathrm{F}<50$ & $F>=50$ & Men & & $\mathrm{F}<50$ & $\mathrm{~F}>=50$ & Men & \\
\hline 4 & $\mathrm{~N}=58$ & $N=67$ & $N=87$ & & $\mathrm{~N}=41$ & $\mathrm{~N}=29$ & $\mathrm{~N}=36$ & & $\mathrm{~N}=17$ & $\mathrm{~N}=38$ & $N=51$ & \\
\hline PAS-positive macrophage, $\%$ & & & & & & & & $*$ & & & & \\
\hline Scattered & 13.2 & 21.7 & 29.7 & & 5.4 & 28.0 & 24.1 & & 31.3 & 17.1 & 33.3 & \\
\hline Clusters & 86.8 & 78.3 & 70.3 & & 94.6 & 72.0 & 75.9 & & 68.8 & 82.9 & 66.7 & \\
\hline Lipogranulomas present,\% & 5.4 & 21.2 & 17.4 & $*$ & 7.7 & 3.6 & 8.6 & & 0.0 & 34.2 & 23.5 & $*$ \\
\hline Copper-stained hepatocytes, \% & & & & & & & & & & & & * \\
\hline
\end{tabular}

This article is protected by copyright. All rights reserved 


\begin{tabular}{|l|c|c|c|c|c|c|c|c|c|c|c|}
\hline Grade 0 & 92.6 & 91.9 & 82.9 & & 89.7 & 92.6 & 93.1 & 100.0 & 91.4 & 76.6 & \\
\hline Grade 1 & 7.4 & 8.1 & 15.8 & & 10.3 & 7.4 & 6.9 & & 0.0 & 8.6 & 21.3 \\
\hline Grade 2 & 0.0 & 0.0 & 1.3 & & 0.0 & 0.0 & 0.0 & 0.0 & 0.0 & 2.1 & \\
\hline
\end{tabular}

Histologic grades were scored in a standardized manner. ${ }^{16} * \mathrm{P}<0.05,{ }^{* *} \mathrm{P}<0.001$ : for the comparison among gender/age categories (Kruskal-Wallis or Chisquare test). Eosinophil infiltration, neutrophil infiltration, central vein endophlebitis, nodular transformation, confluent necrosis, hepatocyte ballooning, cholangiolar cholestasis, ductular reaction, and sinusoidal reticuloendothelial iron stain were not significantly associated with the gender/age categories in any of the above analyses (data not shown).

Table 3a: Associations of the gender/age group categories with histologic features using logistic and ordinal logistic regression models

\begin{tabular}{|c|c|c|c|c|c|c|}
\hline \multirow{3}{*}{ Histologic features } & \multicolumn{4}{|c|}{ Model 1} & \multirow{2}{*}{\multicolumn{2}{|c|}{$\begin{array}{c}\text { Model } 2 \\
\text { Men vs Women(ref) }\end{array}$}} \\
\hline & \multicolumn{2}{|c|}{ Men vs. Women<50(ref) } & \multicolumn{2}{|c|}{ Women $>=50$ vs. Women<50(ref) } & & \\
\hline & Odds ratio $(95 \% \mathrm{Cl})$ & $P$ value & Odds ratio $(95 \% \mathrm{Cl})$ & $P$ value & Odds ratio $(95 \% \mathrm{Cl})$ & $P$ value \\
\hline Interface hepatitis ${ }^{\mathrm{a}^{*}}$ & $0.42(0.22,0.81)$ & 0.009 & $0.51(0.26,1.02)$ & 0.055 & $0.63(0.37,1.05)$ & 0.076 \\
\hline Noticeable plasma cell infiltration $^{b}$ & $0.30(0.13,0.74)$ & 0.009 & $1.15(0.50,2.50)$ & 0.792 & $0.29(0.13,0.62)$ & 0.002 \\
\hline Cholestasis degree $^{b^{*}}$ & $3.63(1.82,7.27)$ & $<.001$ & $0.94(0.46,1.92)$ & 0.868 & $3.76(2.15,6.57)$ & $<.001$ \\
\hline Hepatocellular cholestasis ${ }^{b}$ & $2.85(1.33,6.08)$ & 0.007 & $0.94(0.43,2.04)$ & 0.876 & $2.95(1.58,5.48)$ & $<.001$ \\
\hline Hepatocyte rosettes $^{b}$ & $0.29(0.12,0.66)$ & 0.004 & $0.62(0.27,1.43)$ & 0.260 & $0.37(0.18,0.76)$ & 0.007 \\
\hline Canalicular cholestasis $^{b}$ & $2.36(1.12,4.99)$ & 0.025 & $0.87(0.41,1.84)$ & 0.712 & $2.56(1.38,4.73)$ & 0.003 \\
\hline Lobular disarray $^{\mathrm{b}}$ & $0.36(0.15,0.87)$ & 0.023 & $0.69(0.29,1.66)$ & 0.407 & $0.43(0.20,0.94)$ & 0.033 \\
\hline Iron Satin-hepatocellular iron ${ }^{b}$ & $8.07(3.33,19.53)$ & $<.001$ & $2.53(1.02,6.28)$ & 0.046 & $4.53(2.41,8.54)$ & $<.001$ \\
\hline
\end{tabular}

${ }^{a}$ adjusted for liver injury type, black race and age $\geq 70{ }^{b}$ adjusted for liver injury type, black race.

Model 1 was to assess the associations of histologic features in men and women $>=50$ vs. women $<50$ (reference) while Model 2 was to assess the associations of histologic features in men vs. women (reference). Logistic regression models were used except for the two ordinal variables $(*)$ where proportional odds

This article is protected by copyright. All rights reserved 
models were used. For the two ordinal variables, proportional odds assumption was met (score tests). The variable of cholestatsis degree was analyzed with three ordinal categories of Grade $0,1-2$, and 3 due to better model fitting. The odds ratio presented is either the usual odds ratio or cumulative odds ratio with odds of higher level categories vs. lower level categories. Therefore, an odds ratio of $>1$ represents an increased likelihood of having a worse outcome.

\begin{tabular}{|c|c|c|c|}
\hline \multirow[b]{2}{*}{ Apoptosis } & \multicolumn{2}{|c|}{ Model 1} & \multirow{2}{*}{$\begin{array}{c}\text { Model } 2 \\
\text { Men vs. Women } \\
\text { COR }(95 \% \mathrm{Cl}), \mathrm{p} \text { value }\end{array}$} \\
\hline & $\begin{array}{l}\text { Men vs. Women<50 } \\
\text { COR }(95 \% \mathrm{Cl}) \text {, p value }\end{array}$ & $\begin{array}{c}\text { Women }>=50 \text { vs. Women }<50 \\
\text { COR }(95 \% \mathrm{Cl}), \mathrm{p} \text { value }\end{array}$ & \\
\hline Grade $1-2$ vs. Grade 0 & $0.57(0.19,1.67), 0.303$ & $0.73(0.24,2.20), 0.577$ & $0.70(0.33,1.50), 0.359$ \\
\hline Grade 2 vs. Grade $0-1$ & $0.25(0.11,0.60), 0.002$ & $0.76(0.33,1.76), 0.523$ & $0.29(0.14,0.62), 0.001$ \\
\hline
\end{tabular}

Categories of Apoptosis: Grade 0=none to rare, Grade 1= mild, Grade 2= moderate. COR: cumulative odds ratio. Cumulative logistic regression model was fit to the ordinal variable of apoptosis because the proportional odds assumption was not satisfied. The models were adjusted for biochemical injury type and black race with women of $<50$ as a reference group (Model 1) or women as a reference group (Model 2). The cumulative odds ratio was computed as an odds of higher level categories vs. lower level categories, therefore, an odds ratio of $>1$ represents an increased likelihood of having a worse outcome.

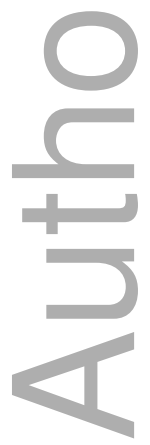

This article is protected by copyright. All rights reserved 